\title{
IN SEARCH OF THE PERFECT HANDSET ANTENNA
}

\author{
Simon P Kingsley ${ }^{(1)}$, David J Ireland ${ }^{(2)}$, Steven G O’Keefe ${ }^{(2)}$, Richard J Langley ${ }^{(3)}$ and Luyi Liu ${ }^{(3)}$ \\ ${ }^{(1)}$ Antenova Ltd.,Far Field House, Albert Road, \\ Stow-cum-Quy, Cambridge, CB25 9AR, UK \\ simon.kingsley@antenova.com \\ ${ }^{(2)}$ Centre for Wireless Monitoring and Applications, Griffith School of Engineering \\ Griffith University, Nathan QLD, Australia 4111 \\ d.ireland@griffith.edu.au \\ s.okeefe@griffith.edu.au \\ ${ }^{(3)}$ Department of Electronic and Electrical Engineering \\ Sheffield University, Mappin Street, SheffieldS1 3JD \\ R.J.Langley@sheffield.ac.uk \\ Luyi.Liu@sheffield.ac.uk
}

\begin{abstract}
The 'perfect antenna' for a handset will be a very low profile module, perhaps measuring $35 \mathrm{x} 10 \mathrm{x} 3$ or $4 \mathrm{~mm}$ and it may well have to share this volume with other components. It will work over a wide range of frequencies from perhaps as low as FM radio bands up to $6 \mathrm{GHz}$ and will exhibit minimal detuning when held in the hand or used next to the head. It will be quickly and easily configured for different devices. There are physical limitations that prevent a simple internal antenna from meeting these requirements but there are various technologies and techniques that may be employed to get as close as possible to this perfect antenna. Here we look at some of these techniques and the tradeoffs to each.
\end{abstract}

\section{Challenges facing the handset antenna designer}

The Industrial Design of modern mobile phones leaves very little space for the antenna and often it must be shared with other components such as cameras and speakers. On many handsets there must be a full groundplane beneath the antenna because the other side of the main PCB is populated with components. The number of antennas used in mobile phones is increasing all the time, see Figure 1. Many of these new applications, such as DVB-H, FM, DAB, NFC, etc., work at lower frequencies than the cellular bands and this makes the antenna design more difficult. Multiple antennas for diversity are also now being introduced requiring even smaller antennas to be located in the handset. Unfortunately, the Chu-Harrington (C-H) limit [1], [2] does not permit antennas to become indefinitely small.

To investigate small antenna limits, a statistical approach has been used at Griffith University, Brisbane, in which a cluster of computing nodes were used to simulate a large number of variations in shape and size of an electrically small antenna. Figure 2(a) shows 3000 plots of antenna volume versus return loss. It can be seen that there are no examples where the antenna volume is small and the return loss large. It is easy to identify the set of optimum results that define the Pareto frontier, see Figure 2(b). These results confirm in a statistical sense that there is a limit to how small a PIFA can be made with a good bandwidth and it would be most interesting to know if this frontier corresponds to the $\mathrm{C}-\mathrm{H}$ limit. A pure dielectric PIFA [3], [4] was chosen for these experiments because there are few loss mechanisms and lower volumes thus tend to result in lower bandwidth rather than lower efficiency. This helps to separate the two problems. Finding the constrained Pareto frontier can be a great help to the antenna designer, as the full set of parameters need not be explored.

When multiple protocols are used on a single handset the first problem is to decide whether a single wide-band antenna should be used or multiple narrower band antennas. A wide-band antenna solution has problems not only with obtaining sufficient bandwidth to cover all the bands but also difficulties associated with the insertion loss, cost, bandwidth and size of the circuits needed to diplex the signals together. Multiple single-band antennas, on the other hand, have problems dominated by the coupling between them and the difficulties of finding sufficient real estate on the handset. Generally, these problems are harder to solve than the single antenna ones. 
Besides the need for wide band operation, modern mobile antennas are usually required to operate over a full groundplane, exhibit minimum detuning, be compatible with different chassis and sometimes use different polarisations (e.g. RHCP for GPS). And of course, they have to be very low cost.

\section{Current solutions}

One way to increase bandwidth in a small patch antenna is to use multi-moding. An example of good design practice here is the Motorola Folded Inverted Conformal Antenna (FICA) [5] and E-patch [6] antennas, see Figure 3(a). Common, differential and slot modes can all be excited in a single structure giving bandwidths up to $30 \%$. A simpler technique is to use groundplane-free monopole designs; although this is not generally popular, it has been used with good effect on the Motorola RAZR and SLVR series of phones see Figure 3(b). Monopoles can have exceptional performance in free space but are prone to detuning when loaded in the talk position. Antenova has pioneered the use of high dielectric antennas (HDA) as a means of obtaining wide bandwidths and improved resistance to detuning, Figure 3(c). Antenova has also developed a series of modules combining the radio with quasi-balanced or complementary antennas in order to address the issues concerning the integration of antennas into handset that were raised in the previous section, see Figure 3(d). This technique has been exploited in a series of complete GPS solutions (antenna, radio and baseband processor) that can be used in many different types of applications just by changing just two components; see Figures 4 (a-c).

Useful as these techniques are, they will not meet our requirements for the perfect antenna. For this we are going to need electronic tuning of the antenna to get round the restrictions of the $\mathrm{C}-\mathrm{H}$ limit.

\section{The high technology antenna}

One of the most promising technologies to be applied to antennas is the development of electronic band gap (EBG) materials. A disadvantage of EBG is limited bandwidth and in fact there is a fundamental thickness to bandwidth ratio limit, similar to the $\mathrm{C}-\mathrm{H}$ limit [7]. To overcome this limitation researchers have begun to investigate the possibility of using active EBG, where tuning elements, such as varactor diodes, can be implemented within the EBG to vary the bandwidth, see [8]. An ideal application might be an EBG that is transparent in the low band, allowing the handset PCB to be driven as part of the antenna, but opaque in the high bands where a variety of balanced antenna structures might then be used. The trade-off is that tuneable EBG structures come at increased cost.

The antenna itself may be tuned by a variety of techniques, the most promising of which is probably using microelectromechanical systems (MEMS) to reconfigure multiband PIFAs. Figure 5(a) shows an example at Sheffield University using MEMS RF switches to control the position of the coupling point between a driven patch and a parasitic element. Figure 5(b) shows the simulated return loss and the tuning effect of changing the switch combination. MEMS RF switches exhibit low-loss, high linearity and good power handling but the disadvantages are the packaging costs and the voltages needed to switch them can be 3-4 times the battery voltage available on a handset.

Antenna tuning may also be accomplished by electronically modifying the nature of the supporting material. Physical changes can be made using electro-active polymers but the most potential is shown by materials with variable dielectric and magnetic properties. With liquid crystals (LC) a reorientation of long polar molecules occurs under the influence of an externally applied electrostatic field and this leads to a change in the dielectric constant. Figure 6(a) shows tunable microstrip antenna in which the molecular orientation can be controlled by applying a DC voltage between the patch and the ground plane. Figure 6(b) shows the simulated return loss and the tuning effect of changing the applied DC voltage. The drawback of LC materials is that they tend to be lossy at cellular frequencies so careful design is needed to use them effectively. Far greater changes can be made with materials such as Barium Strontium Titanate (BST) but the applied voltages needed are much greater too. New materials can also be used for adaptive impedance matching and commercial modules are now available, see [9].

\section{Conclusion}

PIFAs remain one of the best candidates on which to base internal antenna designs because of their low cost, low profile and capability of operating over a full groundplane, but their main limitation is a narrow impedance bandwidth. The first step to improving the PIFA is to design for multimode operation such that the antenna is unbalanced in the low bands and balanced in all the higher bands. 
Electronic tuning of the antenna, the matching circuit and material properties of the supporting structure may all be used to extend the operating band. The biggest advances will be made when tunable EBG materials can be inserted between the antenna and the handset PCB. This will allow more dipole like structures to be used that are inherently efficient and wide band. The final step toward the perfect handset antenna is to include the radio with the antenna in a single pre-tuned module ready for integration into a device. If the same power amplifiers are used in the module then the antenna can be tuned to present the best match to the amplifiers in the talk position. This will ensure the module operates at maximum efficiency under the worst loading conditions.

The advent of software-defined radio will soon allow radio communications over a wide range of frequencies. Reconfigurable antenna technology for mobile platforms will be needed to keep pace with the radio while continuing to meet the ever shrinking space challenges for a smaller antenna. The perfect handset antenna will meet these needs through electronic tuning and closer integration with the $\mathrm{RF}$ front end and the radio.

\section{References}

[1] L. J. Chu, "Physical Limitations of Omni-Directional Antennas", Journal of Applied Physics, Vol. 19, pp. 1163-1175, 1948.

[2] R.C. Hansen, "Fundamental Limitations in Antennas", Proceedings of the IEEE, Vol. 69, No. 2, pp. 170-182, 1981.

[3] Ireland, D. J., Kingsley, S. P. and O'Keefe, S. G.: “A pure dielectric PIFA for WLAN applications", to be presented at Loughborough Antennas and Propagation Conference (LAPC), 2008.

[4] J M Ide, S P Kingsley, S G O'Keefe and S Saario, "Pure dielectric antennas and related devices", UK Patent No 2422248.

[5] Di Nallo, C. and Faraone, A.: "Multiband internal antenna for mobile phones", Electronics Letters 28th April 2005 Vol. 41 No. 9

[6] Caratelli, D., Cicchetti, R., Bit-Babik, G. and Faraone, A.: “A Perturbed E-Shaped Patch Antenna for Wideband WLAN Applications", IEEE Transactions on Antennas and Propagation, Vol. 54, No. 6, June 2006.

[7] Brewitt-Taylor, C. W.: "Limitation on the bandwidth of artificial magnetic conductor surfaces", Loughborough Antennas and Propagation Conference (LAPC), 2006.

[8] Lee, H-J, Ford, K.L. and Langley, R.J.: "Independent multiband antenna tuning using an active EBG", to be presented at Loughborough Antennas and Propagation Conference (LAPC), 2008.

[9] http://www.paratek.com/

\section{Figures}

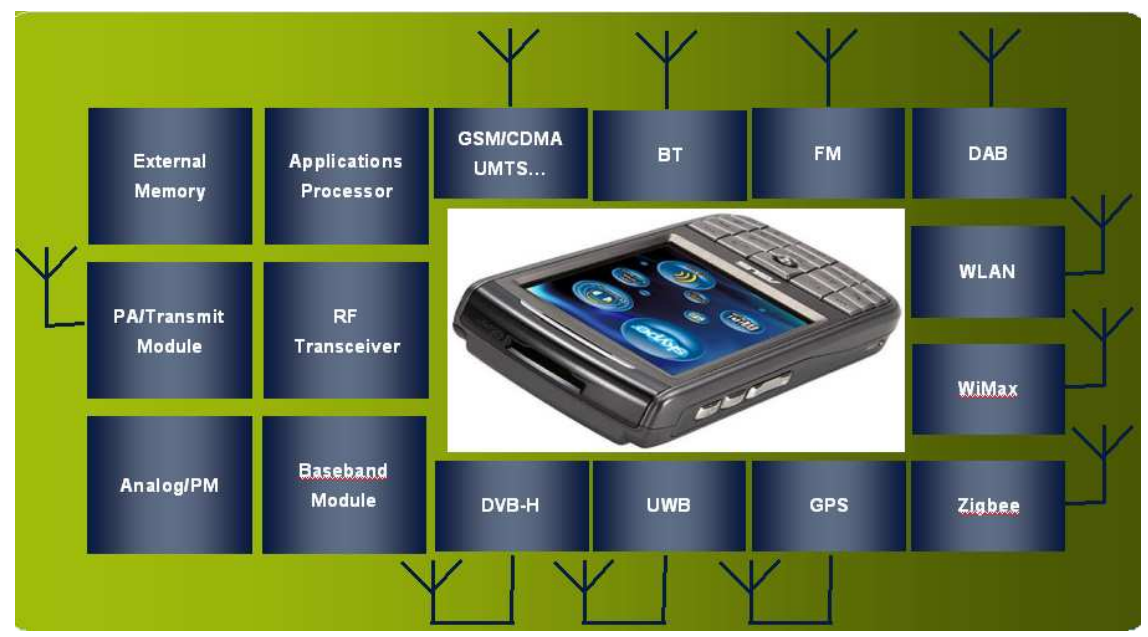

Fig. 1 Some of the protocols requiring antennas on a modern handset. 


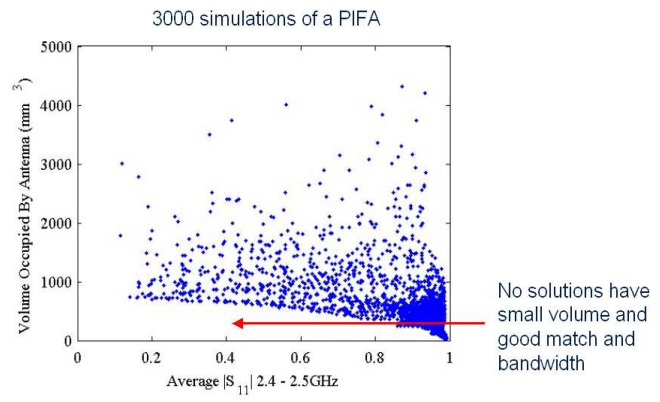

Fig 2 (a) 3000 variations of a pure dielectric PIFA

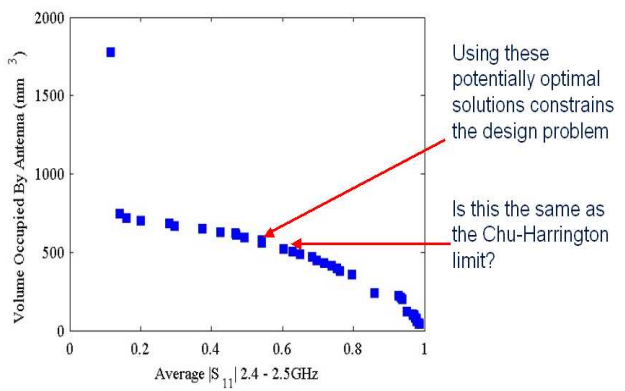

(b) the Pareto frontier

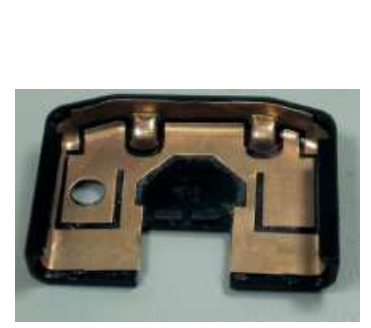

Fig 3 (a) Motorola FICA

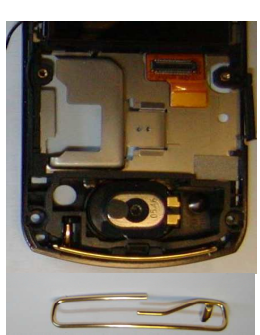

(b) Motorola monopole

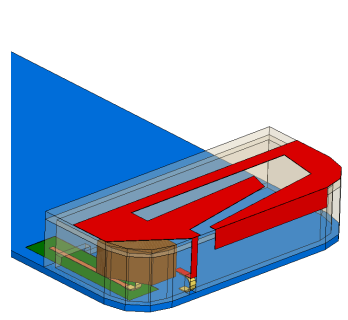

(c) Antenova HDA

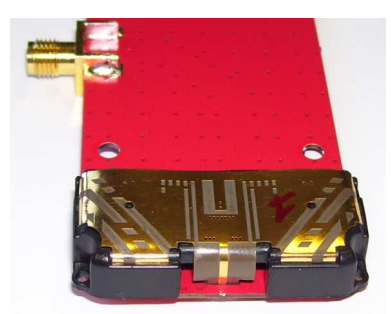

(d)Antenova complementary pair

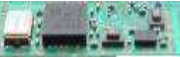

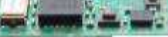

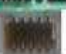

Fig 4 (a) Antenova GPS module

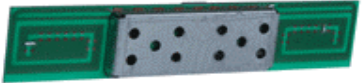

(b) with shielding can in place

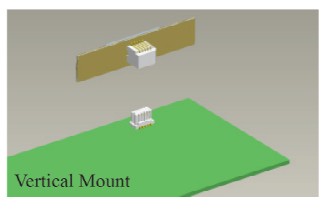

(c) typical application

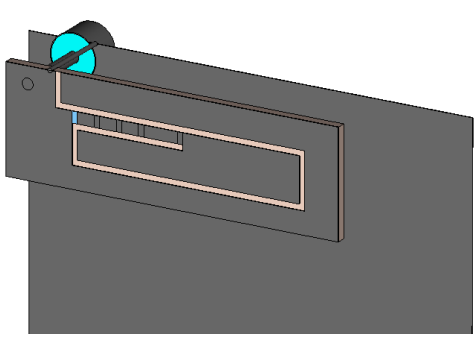

Fig 5 (a) MEMS Reconfigurable Multi-band PIFA

(b) simulated return loss for different switch combinations

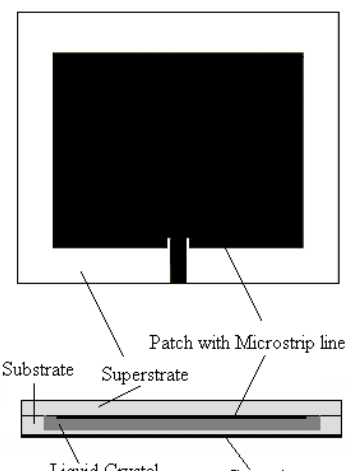

Fig 6 (a) Microstrip patch antenna tuned by LCP

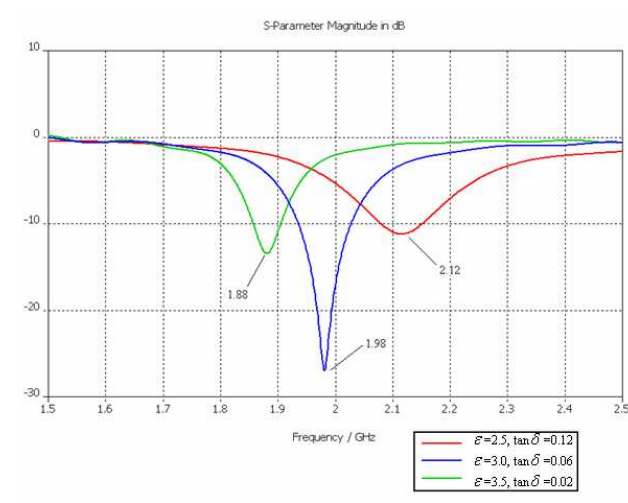

(b) simulated return loss for different applied voltages 